\title{
Involvement of c-Myc in the proliferation of MCF-7 human breast cancer cells induced by bHLH transcription factor DEC2
}

\author{
YUNYAN WU ${ }^{1}$, HIDENOBU SATO ${ }^{1}$, TAKAHIRO SUZUKI ${ }^{1}$, TADASHI YOSHIZAWA ${ }^{1}$, SATOKO MOROHASHI ${ }^{1}$, \\ HIROKO SEINO $^{1}$, TAKESHI KAWAMOTO ${ }^{2}$, KATSUMI FUJIMOTO ${ }^{2}$, YUKIO KATO $^{2}$ and HIROSHI KIJIMA ${ }^{1}$ \\ ${ }^{1}$ Department of Pathology and Bioscience, Hirosaki University Graduate School of Medicine, \\ Hirosaki 036-8562; ${ }^{2}$ Department of Dental and Medical Biochemistry, Hiroshima University \\ Graduate School of Biomedical Sciences, Hiroshima 734-8553, Japan
}

Received May 20, 2014; Accepted November 25, 2014

DOI: $10.3892 /$ ijmm.2014.2042

\begin{abstract}
Differentiated embryonic chondrocyte expressed gene 1 (DEC1; BHLHE40/STRA13/SHARP2) and differentiated embryonic chondrocyte expressed gene 2 (DEC2; BHLHE41/ SHARP1) are basic helix-loop-helix (bHLH) transcriptional factors that are involved in the regulation of cell differentiation, circadian rhythms, response to hypoxia and carcinogenesis. Previous studies have demonstrated that the expression of DECs is induced under hypoxic conditions in various normal and cancer cell lines. In the present study, using RT-qPCR and western blot analysis, we demonstrated that hypoxia induced the expression of DEC1 and DEC2 in MCF-7 human breast cancer cells; their expression levels reached a peak at different time points. In particular, we found that the expression pattern of the hypoxia-inducible factor (HIF)- $1 \alpha$ protein was similar to $\mathrm{DEC} 1$, and that of the HIF-2 $\alpha$ protein was identical to that of DEC2. The knockdown of HIF-2 $\alpha$ using siRNA suppressed the phosphorylation of Akt, as well as the expression of DEC2 and c-Myc. Hypoxia failed to affect the expression of DEC2 and c-Myc when the PI3K/Akt signaling pathway was blocked. In addition, the overexpression of DEC1 and DEC2 was induced by transfecting the cells with a pcDNA vector. The overexpression of DEC2, but not that of DEC1, increased the proliferation of the MCF-7 cells under both normoxic and hypoxic conditions. Concomitantly, the expression of c-Myc was upregulated by
\end{abstract}

Correspondence to: Dr Yunyan Wu, Department of Pathology and Bioscience, Hirosaki University Graduate School of Medicine, 5 Zaifu-cho, Hirosaki 036-8562, Japan

E-mail: yunyanwu@cc.hirosaki-u.ac.jp

Abbreviations: DEC1, differentiated embryonic chondrocyte expressed gene 1; DEC2, differentiated embryonic chondrocyte expressed gene 2

Key words: differentiated embryonic chondrocyte expressed gene 1, differentiated embryonic chondrocyte expressed gene 2, hypoxia, MCF-7 exposure to hypoxia and by the overexpression of DEC2. In conclusion, DEC2 participates in hypoxia-induced cell proliferation by functioning as a target gene of the PI3K/Akt signaling pathway and regulating the expression of c-Myc.

\section{Introduction}

Breast cancer is the most common type of cancer in women worldwide. It has become the second most common cause of cancer-related mortality in women according to the statistical data supplied by Siegel et al in 2013 (1). Although the mortality rate due to breast cancer has declined by approximately $30 \%$ over the past 20 years, metastatic breast cancer remains incurable (1). A low oxygen status (hypoxia or anoxia) is a characteristic of most solid tumors and is associated with malignant progression in several types of cancer, including breast cancer (2). It also severely affects the efficacy of radiotherapy or chemotherapy, as hypoxia usually protects tumor cells from being damaged through several pathways (3-6): i) severe structural and functional abnormalities of tumor microvessels (perfusion-limited $\mathrm{O}_{2}$ delivery), ii) deterioration of diffusion geometry (diffusion-limited $\mathrm{O}_{2}$ delivery) and iii) tumor-associated and/or therapy-induced anemia leading to a reduced $\mathrm{O}_{2}$ transport capacity of the blood (anemic hypoxia) (7). The hypoxia-inducible factor (HIF) transcription factors mediate the primary transcriptional responses to hypoxic stress in normal and transformed cells. An $\mathrm{O}_{2}$-labile $\alpha$-subunit (HIF-1 $\alpha$, HIF- $2 \alpha$ and HIF- $3 \alpha$ ) and a stable $\beta$-subunit (Arnt1, Arnt2 and Arnt3) constitute the heterodimeric HIF proteins. The difference between HIF-1 $\alpha$ and HIF-2 $\alpha$ is mainly observed in the N-terminal transactivation domain (N-TAD), which contributes to target gene specificity, while the C-terminal transactivation domain (C-TAD) is homologous between the isoforms and promotes the expression of their common target genes (8). Recent studies have reported interesting data on distinguishing the roles of HIF-1 $\alpha$ and HIF- $2 \alpha$. Although the two isoforms share some similar properties, they exhibit unique and even opposite characteristics when expressed in the same cell type $(9,10)$. These effects are partly mediated through the regulation of distinct targets, as well as through direct and indirect interactions with complexes that contain important oncoproteins and tumor suppressors. 
As one of the significant oncoproteins, c-Myc has been reported to participate in multiple aspects of cellular function, such as replication, growth, metabolism, differentiation, apoptosis and carcinogenesis (11-16). It activates and represses the transcription of target genes by binding to the E-box (CACGTG) motif after forming a heterodimer with another basic helixloop-helix/leucine zipper (bHLH/Zip) protein MAX. The aberrant expression of c-Myc is closely related to various types of cancer.

Human differentiated embryonic chondrocyte expressed genes (DECs), mouse stimulated by retinoic acid (STRA), and rat split- and hairy-related protein (SHARP) constitute a structurally distinct class of bHLH proteins. DEC2 is an important factor in the regulation of apoptosis, cell differentiation, tumor progression, circadian rhythms and the response to hypoxia. However, the roles of DEC2 under hypoxic conditions have not yet been clarified. The present study demonstrates that DEC2 promotes the proliferation of MCF-7 breast cancer cells by regulating the expression of the c-Myc oncoprotein under hypoxic conditions and through the activation of the PI3K/Akt signaling pathway.

\section{Materials and methods}

Cell culture and treatment. MCF-7 human breast cancer cells were cultured as previously described (17). Hypoxia $\left(3 \% \mathrm{O}_{2}\right)$ was induced by the culture of cells for various periods of time $(2,8$ and $24 \mathrm{~h})$ inside an air-tight chamber with inflow and outflow valves that was infused with a mixture of $3 \% \mathrm{O}_{2}, 5 \%$ $\mathrm{CO}_{2}, 92 \% \mathrm{~N}_{2}$ (BNP-110; ESPEC Corp., Osaka, Japan). In oder to block PI3K, the cells were incubated with the PI3K/Akt inhibitor, LY294002 (Calbiochem, San Diego, CA, USA) at $10 \mu \mathrm{M}$ for $1 \mathrm{~h}$, followed by incubation at $3 \% \mathrm{O}_{2}$ for $24 \mathrm{~h}$.

Reverse transcription-quantitative polymerase chain reaction $(R T-q P C R)$. Three independent RNA samples were prepared from the above cells for RT-qPCR. Total RNA was isolated using an RNeasy RNA Isolation kit (Qiagen $\mathrm{GmbH}$, Hilden, Germany). First-strand cDNA was synthesized from $1 \mu \mathrm{g}$ of total RNA using ReverTra Ace (Toyobo Co., Ltd., Osaka, Japan). Quantitative (real-time) PCR was performed using SYBRGreen Master Mix (Life Technologies, Carlsbad, CA, USA). The sequences and product sizes of the DEC1 (BHLHE40/ STRA13/SHARP2) and DEC2 (BHLHE41/STRA13/SHARP1) primer sets were described in a previous study (18).

DEC1 and DEC2 overexpression. Human DEC1 and DEC2 cDNA were subcloned into pcDNA/zeo as previously described (18). MCF-7 cells were seeded at $5 \times 10^{4}$ cells $/ 35-\mathrm{mm}$ well. DEC1 or DEC2 pcDNA was transfected into the cells using the lipofectamine LTX (Invitrogen, Carlsbad, CA, USA) $24 \mathrm{~h}$ later. Following transfection, the cells were incubated for an additional $24 \mathrm{~h}$ and subjected to western blot analysis.

Short interference RNA (siRNA). siRNA oligos against human HIF- $2 \alpha$ were obtained from Santa Cruz Biotechnology Inc. (Santa Cruz, CA, USA). For the siRNA transfection experiments, the cells were seeded at $5 \times 10^{4}$ cells $/ 35-\mathrm{mm}$ well. The siRNA was transfected into the cells $24 \mathrm{~h}$ later using the lipofectamine RNAiMAX reagent (Invitrogen) according to the manufacturer's instructions. Following transfection, the cells were incubated under normoxic $\left(\mathrm{O}_{2} 21 \%\right)$ or hypoxic $\left(\mathrm{O}_{2} 3 \%\right)$ conditions for a further $24 \mathrm{~h}$ and subjected to western blot analysis.

Western blot analysis. The cells were lysed using M-PER lysis buffer (Thermo Fisher Scientific, Rockford, IL, USA) and the protein concentration was determined using the bicinchoninic acid (BCA) assay. Cell lysates were subjected to SDS-PAGE, and the proteins were transferred onto PVDF membranes (Immobilion P; Millipore, Tokyo, Japan), which were then incubated with antibodies. Bound antibodies were visualized by chemiluminescence using the ECL or ECL Prime Western Blotting Detection system (Amersham Biosciences, Uppsala, Sweden). The experiment was repeated 3 times.

Antibodies. The membranes for western blot analysis were incubated with antibodies specific to DEC1 (1:10,000; Novus Biologicals, Inc. Littleton, CO, USA), DEC2 (1:20,000; H-72X; Santa Cruz Biotechnology, Inc.), HIF-1 $\alpha$ (1:3,000; H-206X; Santa Cruz Biotechnology, Inc.), HIF-2 $\alpha$ (1:3,000; Santa Cruz Biotechnology, Inc.), c-Myc (1:3,000; Epitomics, Inc., Burlingame, CA, USA), phospho-Akt (1:6,000; Epitomics Inc.), Akt (1:10,000; Epitomics Inc.) and actin (1:20,000; Sigma, St. Louis, MO, USA), followed by horseradish peroxidaseconjugated secondary antibody (IBL, Fujioka, Gunma, Japan). Can Get Signal Immunoreactions Enhancer Solution (Toyobo Co., Ltd.) or Immunoshot Immunoreaction Enhancer Solution (Cosmobio Co., Ltd., Tokyo, Japan) was used to dilute the primary antibodies.

Cell proliferation assay. Cell proliferation was measured by 3-(4,5-dimethylthiazol-2-yl)-5-(3-carboxymethoxyphenyl)-

2-(4-sulfophenyl)-2H-tetrazolium (MTS) assay. The MCF-7 cells were seeded in 96-well plates. The cells were transfected with an empty plasmid (pcDNA) or the DEC1 or DEC2 expression plasmid. After $18 \mathrm{~h}$ of transfection, the cells were cultured under hypoxic conditions for a further $24 \mathrm{~h}$. Subsequently, the cells were added to each well along with the CellTiter $96^{\circledR}$ $\mathrm{AQ}_{\text {ueous }}$ One Solution Reagent (Promega Corp., Madison, WI, USA) and were incubated at $37^{\circ} \mathrm{C}$ for an additional $1 \mathrm{~h}$. Absorbance $\left(\mathrm{OD}_{490} \mathrm{~nm}\right)$ was measured using a 96-well plate reader.

Statistical analysis. The results are presented as the means \pm standard error of the mean (SEM) of the number of experiments indicated in the figure legends. Statistical analysis was performed using the Student's t-test. The level of statistically significant differences was set at $\mathrm{P}<0.05$, and the level of highly significant differences at $\mathrm{P}<0.001$.

\section{Results}

Effects of exposure to hypoxia on the expression of DECI and DEC2 in MCF-7 cells. Firstly, we analyzed the effects of exposure to hypoxia on gene expression in MCF-7 human breast cancer cells. As shown in Fig. 1, exposure of the cells to $\mathrm{O}_{2}(3 \%)$ for 2,8 and $24 \mathrm{~h}$ induced the protein expression of HIF-1 $\alpha$ and HIF-2 $\alpha$ when compared with the control cells exposed to normal oxygen conditions. In addition, HIF-1 $\alpha$ expression 


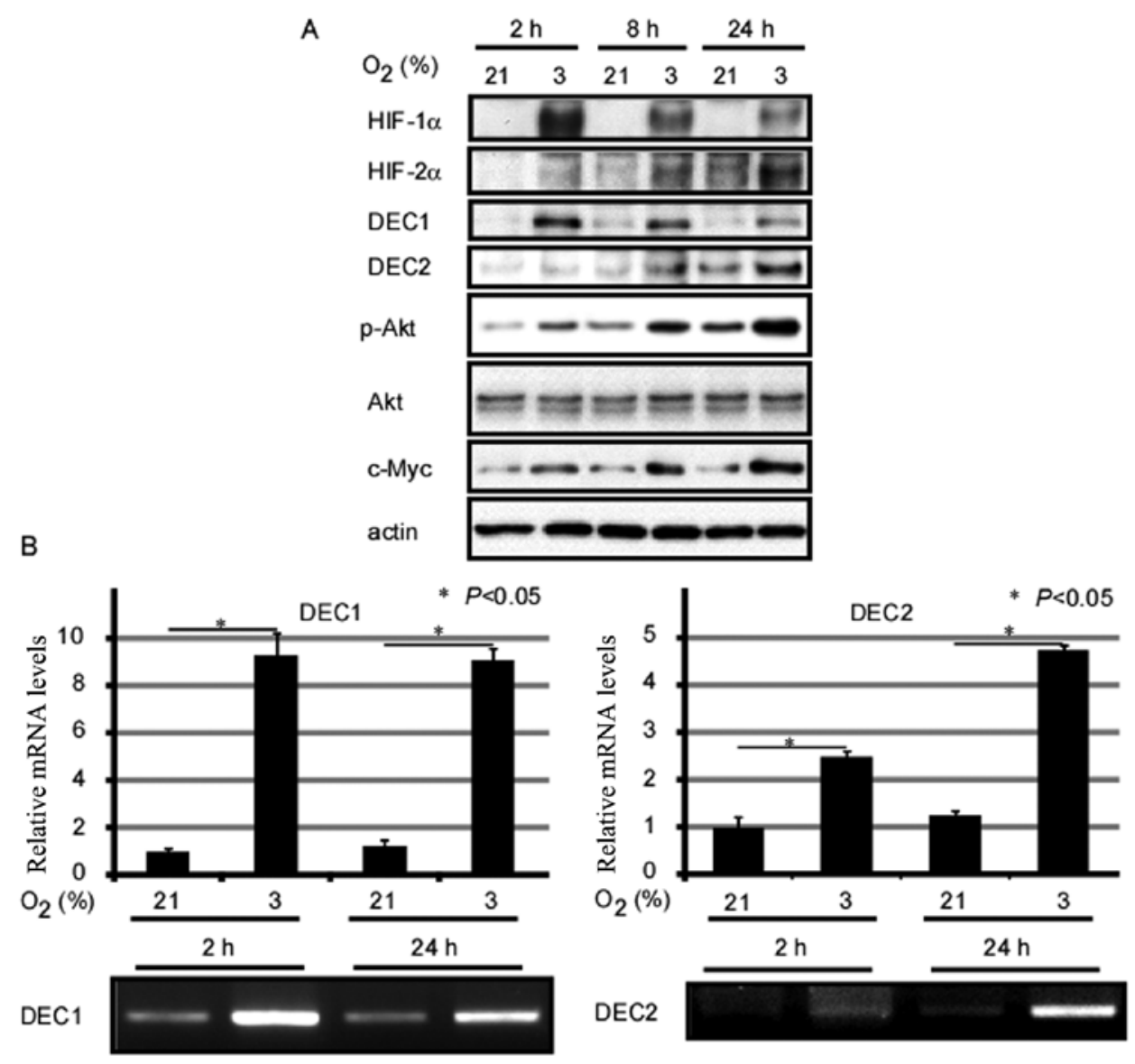

Figure 1. Exposure to hypoxia upregulates the expression of differentiated embryonic chondrocyte expressed gene (DEC) 1 and DEC2 in MCF-7 breast cancer cells. (A) MCF-7 cells were cultured under hypoxic conditions for 2, 8 and $24 \mathrm{~h}$. Cell lysates were prepared and subjected to western blot analysis for the expression of hypoxia-inducible factor (HIF)-1 $\alpha$, HIF-2 $\alpha$, DEC1, DEC2, phosphorylated (p-)Akt, Akt, c-Myc and actin. Representative data of 3 independent experiments with similar results are shown. (B) MCF-7 cells were exposed to hypoxia $\left(\mathrm{O}_{2} 3 \%\right)$ or normoxia $\left(\mathrm{O}_{2} 21 \%\right)$ for 2 and $24 \mathrm{~h}$. Total RNA was prepared and subjected to RT-qPCR for the mRNA expression of DEC1 and DEC2. Each value represents the mean \pm SEM (bars) of 3 independent experiments ("P<0.05, according to the t-test).

levels reached a peak at $2 \mathrm{~h}$, whereas HIF- $2 \alpha$ expression levels reached a peak at $24 \mathrm{~h}$. Although the endogenous expression of DEC1 and DEC2 was weak in the MCF-7 cells, their mRNA and protein expression levels markedly increased under hypoxic conditions. Furthermore, the DEC1 protein level reached its peak when the cells were incubated under hypoxic conditions for $2 \mathrm{~h}$, whereas the DEC2 protein expression level reached its peak following the exposure of the cells to hypoxia for $24 \mathrm{~h}$. However, the relative mRNA expression of DEC1 showed no significant change between the 2- and 24-h time points following exposure to hypoxia. Moreover, exposure to hypoxia induced the expression of c-Myc and the phosphorylation of Akt in the MCF-7 cells (Fig. 1).

Exposure to hypoxia for $24 \mathrm{~h}$ enhances the proliferation of MCF-7 cells. Both Akt phosphorylation and c-Myc upregulation are closely associated with the proliferation of various types of cancer cells $(14,19)$. Thus, we examined whether exposure to hypoxia affects the proliferation of MCF-7 human breast cancer cells by MTS assay (Fig. 2A). Exposure to hypoxia for $24 \mathrm{~h}$ induced the proliferation of the MCF-7 cells, and the DEC2-overexpressing MCF-7 cells showed an increased proliferation rate when compared with either the
pcDNA-transfected- or the DEC1 pcDNA-transfected cells. Moreover, DEC2 overexpression enhanced the proliferation of the MCF-7 cells under both normoxic and hypoxic conditions.

Overexpression of DEC2 induces c-Myc expression in MCF-7 cells. To clarify the biological functions of DEC1 and DEC2 in breast cancer cells, we transiently transfected the expression vectors for DEC1 or DEC2 into the MCF-7 cells and analyzed whether DEC1 or DEC2 affects the expression of c-Myc. Under normoxic conditions, c-Myc expression was slightly induced by the overexpression of DEC2; however, the c-Myc protein level was sharply elevated when DEC2 was overexpressed under hypoxic conditions. By contrast, DEC1 overexpression under both normoxic and hypoxic conditions did not affect the expression levels of c-Myc when compared with the empty vector-transfected cells (Fig. 2B). The above data suggest that $\mathrm{DEC} 2$, but not DEC1, regulates the expression of $\mathrm{c}-\mathrm{Myc}$ in MCF-7 cells.

Knockdown of HIF-2 $\alpha$ inhibits Akt phosphorylation, as well as the expression of DEC 2 and $c-M y c$. It has been reported that HIF- $2 \alpha$ induces the proliferation of renal cell carcinoma cells (20). We thus hypothesized that HIF-2 $\alpha$ may contribute 

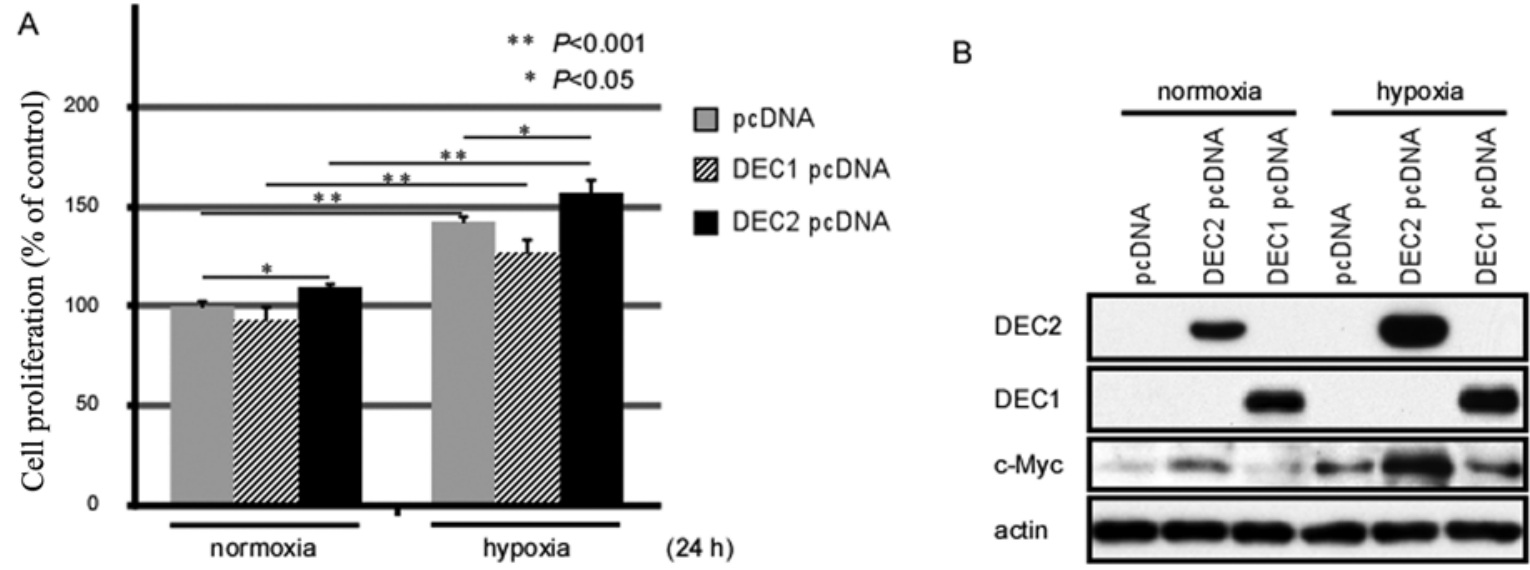

Figure 2. Differentiated embryonic chondrocyte expressed gene (DEC)2 overexpression induces cell proliferation through the upregulation of c-Myc. (A) The enforced expression of DEC2 increased MCF-7 cell proliferation. MCF-7 cells were transfected with pcDNA, DEC1 pcDNA or DEC2 pcDNA and then exposed to hypoxia or normoxia for $24 \mathrm{~h}$, and cell proliferation was measured by MTS assay. The values are shown as a percentage of the control. Each value represents the mean \pm SEM (bars) of 3 independent experiments ( $\mathrm{P}<0.05$ or ${ }^{* *} \mathrm{P}<0.001$, compared with the control pcDNA). (B) DEC 2 overexpression upregulated the expression of the oncoprotein c-Myc. MCF-7 cells were treated as described above, and cell lysates were prepared from the cells and subjected to western blot analysis for the expression of DEC1, DEC2, c-Myc and actin. One representative of at least 3 independent experiments with similar results is shown.

A

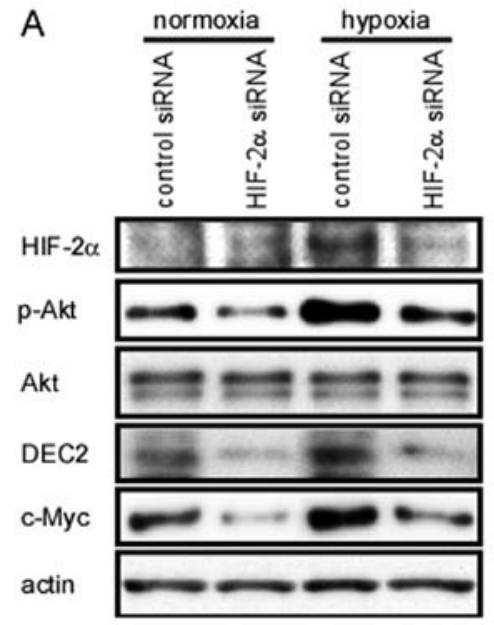

Figure 3. Hypoxia-inducible factor (HIF)-2 $\alpha$ regulates differentiated embryonic chondrocyte expressed gene (DEC) 2 and c-Myc expression by activating Akt. (A) MCF-7 cells were transfected with HIF-2 $\alpha$ short interference RNA (siRNA) and incubated for $24 \mathrm{~h}$. Subsequently, the cells were incubated under hypoxic or normoxic conditions for an additional $24 \mathrm{~h}$, before being lysed. The lysates were subjected to western blot analysis for the expression of HIF-2 $\alpha$, pAkt, Akt, DEC2, c-Myc and actin. (B) MCF-7 cells were incubated with LY294002 (PI3K/Akt inhibitor; $10 \mu \mathrm{M}$ ) for $1 \mathrm{~h}$ before being exposed to hypoxia for $24 \mathrm{~h}$. The lysates were prepared and subjected to western blot analysis for the expression of HIF-2 $\alpha$, pAkt, Akt, DEC2, c-Myc and actin.

to the increased proliferation of MCF-7 cells under hypoxic conditions. In order to characterize the function of HIF-2 $\alpha$ protein, the MCF-7 cells were transiently transfected with siRNA against HIF- $2 \alpha$ for $24 \mathrm{~h}$, followed by another $24 \mathrm{~h}$ of exposure to hypoxia or normoxia; western blot analysis was then performed. The silencing of HIF-2 $\alpha$ suppressed Akt phosphorylation, as well as DEC2 and c-Myc protein expression, regardless of the oxygen concentration (Fig. 3A). However, the expression of DEC1 was not affected by HIF- $2 \alpha$ siRNA (data not shown).

Inhibition of Akt phosphorylation decreases the induction of DEC2 by exposure to hypoxia. DEC1 has been reported to be involved in the phosphorylation of Akt (21). Therefore, we examined the association between Akt and DEC2. Pre-treatment of the MCF-7 cells with LY294002, an inhibitor of the PI3K/Akt signaling pathway, abrogated the hypoxia-induced Akt phosphorylation, as well as DEC2 expression (Fig. 3B). In addition, exposure to hypoxia failed to upregulate the protein level of c-Myc when the PI3K/Akt signaling was blocked.

\section{Discussion}

In the present study, we demonstrate that DEC2 plays a role, at least in part, in the proliferation of MCF-7 breast cancer cells induced by HIF- $2 \alpha$ under low oxygen conditions. Silencing the expression of HIF-2 $\alpha$ inhibited the phosphorylation of Akt, as well as the expression of DEC2 and c-Myc. It seems that DEC2 functions as an effector of the PI3K/Akt signaling pathway, since the interruption of this pathway blocked the induction of DEC2 expression following exposure to hypoxia. In addition, our results demonstrated that DEC2, but not DEC1, upregulated the expression of c-Myc. DEC2 and DEC1 are both transcription factors and have been shown to transrepress their target genes by binding to the E-box elements (22-24). Thus, it is suggested that the regulation of c-Myc by DEC2 may not be mediated through binding to the E-boxes. Future studies are required to clarify the details of the mechanisms through which DEC2 regulates c-Myc expression, and the differences between DEC2 and DEC1 in regulating their target genes.

Although no significant changes in the mRNA level of DEC1 were observed between the cells exposed to hypoxia for 2 and those exposed for $24 \mathrm{~h}$, the protein expression of DEC1 was significantly reduced in the cells cultured under hypoxic conditions for $24 \mathrm{~h}$ compared with that in the cells cultured under hypoxic conditions for 2 and $8 \mathrm{~h}$. DEC 1 has been reported as a marker of tumor hypoxia in the A549 lung adenocarcinoma cell line (25). In that study, the mRNA and 


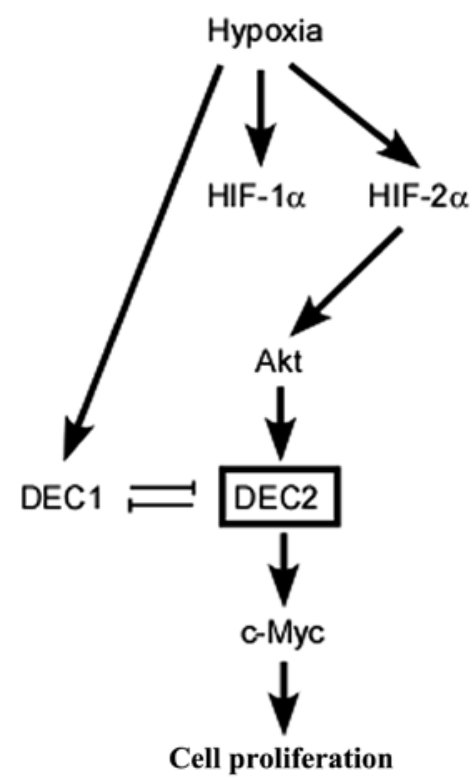

Figure 4. Role of differentiated embryonic chondrocyte expressed gene (DEC) 2 in the hypoxia-induced proliferation of MCF-7 cells. Exposure to hypoxia upregulated the expression of hypoxia-inducible factor (HIF)- $2 \alpha$, which activated the phosphorylation of Akt. DEC2 functioned as a target of the PI3K/Akt pathway and regulated the expression of c-Myc and contributed to the hypoxia-induced proliferation of human breast cancer cells.

protein expression levels of DEC1, as well as those of HIF-1 $\alpha$, were upregulated by hypoxia in a time-dependent manner, and the expression levels reached their peak at the time point of $48 \mathrm{~h}$ (25). However, in our study, the protein expression of DEC1 decreased after the cells were cultured under hypoxic conditions for $24 \mathrm{~h}$, when compared with those cultured for $2 \mathrm{~h}$; however, the mRNA expression level of DEC1 remained stable at all time points. We hypothesized that the differences may arise from the specificity of the cell line and the different methods used for detecting the expression of the protein. In the other studies mentioned above, the expression of DEC1 and HIF-1 $\alpha$ was detected by immunocytochemical staining. Moreover, previous studies have demonstrated that DEC2 and DEC1 inhibit each other by binding the E-box on their promoters (23). Therefore, the reverse expression patterns observed under hypoxic conditions may be partly caused by the inhibitory effects exerted on each other.

DEC expression can be induced by a variety of other stimuli, such as transforming growth factor (TGF) $-\beta$, cytokines, retinoic acid, insulin and light (26-30). In the present study, we found that exposure of the cells to hypoxia increased the expression of DEC1 and DEC2 in a different manner. More specifically, $\mathrm{DEC} 1$ expression reached its peak at $2 \mathrm{~h}$ following exposure to hypoxia, whereas DEC2 expression reached its peak at $24 \mathrm{~h}$ following exposure to hypoxia. We also observed that DEC1 had an expression pattern similar to HIF- $1 \alpha$ protein, whereas DEC2 had an expression pattern similar to that of HIF- $2 \alpha$ protein. Although DEC1 and DEC2 exhibit a similar structure in their $\mathrm{N}$-terminal, the $\mathrm{C}$-terminal sequences are quite different. That is, the proline-rich region of DEC 1 is replaced by the alanine/ glycine-rich domain in DEC2 protein (31). Existing evidence suggests that DEC1 and DEC2 are expressed and function in a different manner during physiological and pathological processes (31). In the present study, we proved that DEC2, but not DEC1, induced cell proliferation through the upregulation of c-Myc expression. Further studies are required to elucidate the function of the C-terminal of DEC1 and DEC2.

As an adverse prognostic factor, independent of standard prognostic factors, such as tumor stage and nodal status, hypoxia has been observed in a wide range of malignancies, including cancer of the breast, the uterine cervix, the rectum, the pancreas, the lungs, the brain, as well as soft tissue sarcomas, non-Hodgkin's lymphomas, malignant melanomas, metastatic liver tumors and renal cell cancer (7). The hypoxic microenvironment of tumors contributes to malignant progression, poor prognosis and resistance to chemotherapy and radiation. HIFs are crucial regulators of the response to low oxygen conditions (7). To date, HIF-1 $\alpha$ and HIF-2 $\alpha$ are the most extensively studied among the HIF isoforms. While the two isoforms share similar domain structures and overlapping target genes, it is now clear that they also have unique target genes. Moreover, HIF-1 $\alpha$ and HIF-2 $\alpha$ usually have divergent roles even in regulating the same genes (32). Recently, it has been reported that HIF-1 $\alpha$ is activated during the initial response to hypoxia, whereas HIF-2 $\alpha$ plays a major role during exposure to chronic hypoxia (33). The similar expression patterns of DEC 2 and HIF-2 $\alpha$ demonstrated in our study suggests that DEC2 is an important mediator during exposure to chronic hypoxia.

The $c-M y c$ gene, which was first described by Bishop et al in 1982, is a bHLH/Zip-type transcription factor (34). c-Myc forms a heterodimer with the bHLH/Zip protein MAX. Following dimerization, this complex binds to specific DNA sites, at CACGTG sequences known as the E-box motif, to activate and repress the transcription of target genes, as well as to modulate chromatin (35). As one of the major human oncogenes, c-Myc is frequently altered in many forms of cancer (35-38). It modulates the cell cycle and cell proliferation, increases cell metabolism and stimulates differentiation, among its many other biological functions. Studies have produced conflicting results as to how HIF-2 $\alpha$ affects c-Myc expression; some researchers have demonstrated that HIF- $2 \alpha$ inhibits c-Myc through direct interaction under physiological conditions $(20,39,40)$. However, others have suggested that HIF-2 $\alpha$ enhances c-Myc activity by binding and stabilizing c-Myc/Max complexes (32). Our results are in agreement with the latter, as we demonstrated that DEC2 regulated c-Myc expression through a yet undetermined mechanism.

Fig. 4 summarizes the possible pathways responsible for the proliferation of MCF-7 breast cancer cells induced by exposure to hypoxia. Although the amino acid sequences of DEC1 and DEC2 share an approximately $40 \%$ homology, they have different effects on the cell proliferation induced by hypoxia and on apoptosis, as previously reported (17). In the present study, we found the c-Myc expression was increased by DEC2 overexpression in the MCF-7 cells. In general, DEC2 is thought to function as a transcriptional repressor by binding to the E-box in the promoters of its target genes (31). In our study, however, DEC2 was shown to function as a positive inducer by enhancing the expression of c-Myc. In our previous study, we demonstrated that siRNA against DEC2 decreased the mRNA level of c-Myc in MCF-7 cells (18). We deduced 
that DEC2 regulated the expression of c-Myc, not only at the transcriptional level, but also at the post-transcriptional level. DEC1 and DEC2 have also been reported to play distinct roles in the epithelial-mesenchymal transition of pancreatic carcinoma cells (41). The differential protein structure and subcellular localization of DEC1 and DEC2 may contribute to the divergent roles in regulating target genes. Undoubtedly, further investigation is warranted to determine the detailed mechanisms through which DEC2 regulates c-Myc expression.

\section{Acknowledgements}

The present study was supported by Grants-in-Aid for Science from the Ministry of Education, Culture, Sports, Science, and Technology of Japan, a Grant for Hirosaki University Institutional Research and the Fund for the Promotion of International Scientific Research.

\section{References}

1. Siegel R, Naishadham D and Jemal A: Cancer statistics, 2013 CA Cancer J Clin 63: 11-30, 2013.

2. Favaro E, Lord S, Harris AL and Buffa FM: Gene expression and hypoxia in breast cancer. Genome Med 3: 55, 2011.

3. Fyles A, Milosevic M, Hedley D, et al: Tumor hypoxia has independent predictor impact only in patients with node-negative cervix cancer. J Clin Oncol 20: 680-687, 2002.

4. Hockel M, Schlenger K, Aral B, Mitze M, Schaffer U and Vaupel P: Association between tumor hypoxia and malignant progression in advanced cancer of the uterine cervix. Cancer Res 56: 4509-4515, 1996.

5. Jubb AM, Buffa FM and Harris AL: Assessment of tumour hypoxia for prediction of response to therapy and cancer prognosis. J Cell Mol Med 14: 18-29, 2010.

6. Nordsmark M, Bentzen SM, Rudat V, et al: Prognostic value of tumor oxygenation in 397 head and neck tumors after primary radiation therapy. An international multi-center study. Radiother Oncol 77: 18-24, 2005.

7. Vaupel $P$ and Mayer A: Hypoxia in cancer: significance and impact on clinical outcome. Cancer Metastasis Rev 26: 225-239, 2007.

8. Hu CJ, Sataur A, Wang L, Chen H and Simon MC: The $\mathrm{N}$-terminal transactivation domain confers target gene specificity of hypoxia-inducible factors HIF-1alpha and HIF-2alpha. Mol Biol Cell 18: 4528-4542, 2007.

9. Keith B, Johnson RS and Simon MC: HIF1 $\alpha$ and HIF2 $\alpha$ : sibling rivalry in hypoxic tumour growth and progression. Nat Rev Cancer 12: 9-22, 2012.

10. Loboda A, Jozkowicz A and Dulak J: HIF-1 versus HIF-2--is one more important than the other? Vascul Pharmacol 56: 245-251, 2012.

11. Packham G and Cleveland JL: c-Myc and apoptosis. Biochim Biophys Acta 1242: 11-28, 1995.

12. Hoffman B and Liebermann DA: The proto-oncogene c-myc and apoptosis. Oncogene 17: 3351-3357, 1998.

13. Dang CV: c-Myc target genes involved in cell growth, apoptosis, and metabolism. Mol Cell Biol 19: 1-11, 1999.

14. Dang CV, Resar LM, Emison E, et al: Function of the c-Myc oncogenic transcription factor. Exp Cell Res 253: 63-77, 1999.

15. Elend $\mathbf{M}$ and Eilers $M$ : Cell growth: downstream of Myc - to grow or to cycle? Curr Biol 9: R936-R938, 1999.

16. Prendergast GC: Mechanisms of apoptosis by c-Myc. Oncogene 18: 2967-2987, 1999.

17. Wu Y, Sato F, Bhawal UK, et al: Basic helix-loop-helix transcription factors DEC1 and DEC2 regulate the paclitaxel-induced apoptotic pathway of MCF-7 human breast cancer cells. Int J Mol Med 27: 491-495, 2011.

18. Liu Y, Sato F, Kawamoto T, et al: Anti-apoptotic effect of the basic helix-loop-helix (bHLH) transcription factor DEC2 in human breast cancer cells. Genes Cells 15: 315-325, 2010.
19. Liang J, Zubovitz J, Petrocelli T, et al: PKB/Akt phosphorylates p27, impairs nuclear import of p27 and opposes p27-mediated G1 arrest. Nat Med 8: 1153-1160, 2002.

20. Gordan JD, Thompson CB and Simon MC: HIF and c-Myc: sibling rivals for control of cancer cell metabolism and proliferation. Cancer Cell 12: 108-113, 2007.

21. Bhawal UK, Sato F, Arakawa Y, et al: Basic helix-loop-helix transcription factor DEC1 negatively regulates cyclin D1. J Pathol 224: 420-429, 2011.

22. Hamaguchi H, Fujimoto K, Kawamoto T, et al: Expression of the gene for Dec2, a basic helix-loop-helix transcription factor, is regulated by a molecular clock system. Biochem J 382: 43-50, 2004.

23. Fujimoto K, Hamaguchi H, Hashiba T, et al: Transcriptional repression by the basic helix-loop-helix protein Dec2: multiple mechanisms through E-box elements. Int J Mol Med 19: 925-932, 2007.

24. Nakashima A, Kawamoto T, Honda KK, et al: DEC1 modulates the circadian phase of clock gene expression. Mol Cell Biol 28: 4080-4092, 2008.

25. Zhang L and Li QQ: Embryo-chondrocyte expressed gene 1, downregulating hypoxia-inducible factor 1alpha, is another marker of lung tumor hypoxia. Acta Pharmacol Sin 28: 549-558, 2007.

26. Zawel L, Yu J, Torrance CJ, et al: DEC1 is a downstream target of TGF-beta with sequence-specific transcriptional repressor activities. Proc Natl Acad Sci USA 99: 2848-2853, 2002.

27. Ivanova AV, Ivanov SV, Zhang X, Ivanov VN, Timofeeva OA and Lerman MI: STRA13 interacts with STAT3 and modulates transcription of STAT3-dependent targets. J Mol Biol 340: 641-653, 2004.

28. Boudjelal M, Taneja R, Matsubara S, Bouillet P, Dolle P and Chambon P: Overexpression of Stra13, a novel retinoic acid-inducible gene of the basic helix-loop-helix family, inhibits mesodermal and promotes neuronal differentiation of P19 cells. Genes Dev 11: 2052-2065, 1997.

29. Yamada K, Kawata H, Shou Z, Mizutani T, Noguchi T and Miyamoto K: Insulin induces the expression of the SHARP-2/Stra13/DEC1 gene via a phosphoinositide 3-kinase pathway. J Biol Chem 278: 30719-30724, 2003.

30. Honma S, Kawamoto T, Takagi Y, et al: Decl and Dec2 are regulators of the mammalian molecular clock. Nature 419: 841-844, 2002.

31. Yamada K and Miyamoto K: Basic helix-loop-helix transcription factors, BHLHB2 and BHLHB3; their gene expressions are regulated by multiple extracellular stimuli. Front Biosci 10: 3151-3171, 2005 .

32. Loboda A, Jozkowicz A and Dulak J: HIF-1 and HIF-2 transcription factors - similar but not identical. Mol Cells 29: 435-442, 2010.

33. Koh MY and Powis G: Passing the baton: the HIF switch. Trends Biochem Sci 37: 364-372, 2012.

34. Bishop JM: Retroviruses and cancer genes. Adv Cancer Res 37: $1-32,1982$.

35. Liao DJ and Dickson RB: c-Myc in breast cancer. Endocr Relat Cancer 7: 143-164, 2000.

36. Weng W, Yang Q, Huang M, et al: c-Myc inhibits TP53INP1 expression via promoter methylation in esophageal carcinoma. Biochem Biophys Res Commun 405: 278-284, 2011.

37. Dang CV, O'Donnell KA, Zeller KI, Nguyen T, Osthus RC and Li F: The c-Myc target gene network. Semin Cancer Biol 16: 253-264, 2006

38. Adhikary S and Eilers M: Transcriptional regulation and transformation by Myc proteins. Nat Rev Mol Cell Biol 6: 635-645, 2005.

39. Zhang H, Gao P, Fukuda R, et al: HIF-1 inhibits mitochondrial biogenesis and cellular respiration in VHL-deficient renal cell carcinoma by repression of C-MYC activity. Cancer Cell 11: 407-420, 2007.

40. Corn PG, Ricci MS, Scata KA, et al: Mxil is induced by hypoxia in a HIF-1-dependent manner and protects cells from c-Myc-induced apoptosis. Cancer Biol Ther 4: 1285-1294, 2005.

41. $\mathrm{Wu} \mathrm{Y}$, Sato F, Yamada T, et al: The BHLH transcription factor DEC1 plays an important role in the epithelial-mesenchymal transition of pancreatic cancer. Int J Oncol 41: 1337-1346, 2012. 